\title{
Impact of The HEROES Project on First Responders' Well-Being
}

\author{
Daniel M. Blumberg, ${ }^{\star}$ Luciano Giromini, ${ }^{\dagger}$ Konstantinos Papazoglou, ${ }^{\ddagger}$ and A. Renee Thornton $\S$
}

\begin{abstract}
First responders experience a myriad of stressors (e.g., operational, organizational, personal) over the course of their career. An abundance of empirical evidence shows that the impact of those stressors on first responders' health, well-being, and performance can be detrimental. Nevertheless, previous research has mainly focused on the role of a specific technique (e.g., mindfulness, breathing exercises, psychoeducation) towards the promotion of well-being among first responders. This allows us to explore the role of a single technique in supporting first responders. However, given the complexity of stressors experienced by this population, it appears that a synergistic role of multileveled intervention is imperative to promote lasting improvement in first responders' well-being. To this end, The HEROES Project, an eight-week online training program, was developed to address the aforementioned gap in the literature. The HEROES Project incorporates lessons that aim to build a cluster of skills that together promote first responders' wellbeing. In the present study, a sample of first responders $(n=124)$ from the US Midwest were recruited and completed The HEROES Project. They were assessed before and after completion of the program, and then follow-up measurements were obtained for two years following the baseline assessment. Results showed that participants with higher distress and lower psychological resources before the training benefited most from The HEROES Project, but that the training significantly improved psychological capital and reduced stress, depression, anxiety, and trauma symptoms for all participants. Clinical and training implications as well as future research directions are discussed.
\end{abstract}

Key Words First responders; well-being; health; performance; resilience; police training.

Journal of CSWB. 2020 April;5(1):8-14

www.journalcswb.ca

\section{INTRODUCTION}

This paper describes the results of a pilot study of a six-lesson, eight-week online wellbeing program for first responders. There is no argument that law enforcement personnel and firefighters experience numerous occupational and organizational stressors, which can jeopardize their health and well-being (e.g., Carpenter et al., 2015; Tuttle, Blumberg, \& Papazoglou, 2019). The threats to well-being affect first responders physically, mentally, emotionally, socially, and spiritually (e.g., Papazoglou \& Blumberg, 2020). Perhaps most alarmingly, in one sample of police officers, over $12 \%$ reported a likelihood of attempting suicide at some point in the future (Thoen, Dodson, Manzo, Piña-Watson, \& TrejosCastillo, 2019). Although suicide represents the most extreme outcome, most first responders experience a range of other well-being-related effects (Mumford, Taylor, \& Kubu, 2015). Clearly, first responders' well-being is crucial for their own and the public's safety (Creighton \& Blumberg, 2016).
Specific efforts to improve first responders' well-being have produced generally positive results. For example, social support was found to reduce a sample of firefighters' occupational stress levels and their suicidal ideation (Carpenter et al., 2015). Likewise, police officers who felt support from their agency reported greater well-being and less stress (Thoen et al., 2019). Resilience training improved a sample of female police officers' psychological well-being and reduced their occupational stress levels (Chitra \& Karunanidhi, 2018). Firefighters' mindfulness was associated with better health outcomes and psychological functioning (Smith et al., 2011). However, questions have been raised about the extent to which employees will use available well-being services (Biebel, 2010; Kuehl, Mabry, Elliot, Kuehl, \& Favorite, 2013) and what sorts of incentives might be necessary to increase employees' use of an online well-being program (Hibbard \& Greene, 2014).

An important question is whether or not the effects of a well-being program will last. One study (Christopher et al., 
2018) demonstrated the very positive impact of mindfulness training to increase resilience among a sample of law enforcement officers. The program showed significant improvement in a variety of health and behavioral outcomes for participants who took part in the training over a control group, but those improvements were not maintained at a three-month followup assessment (Christopher et al., 2018). Similarly, in a recent large study of non-first responders, a workplace well-being program was effective for improving exercise and weight management for participants over the control group members, but "did not generate differences in clinical measures of health, health care spending or utilization, or employment outcomes after 18 months" (Song \& Baicker, 2019, p. 1498).

A possible problem with the longevity of positive effects of many well-being programs is their singular training focus. Many of the above-mentioned studies taught mindfulness, resilience, tactical breathing, or techniques to improve fitness and nutrition. These efforts may be enhanced when there is a concurrent sense of support from the organization, but the benefits may be fleeting when those who followed the program are not sufficiently motivated to continue to practice what was initially taught. This raises questions about the method of delivery of training, the extent to which the organization reinforces the training after it occurs, and, even, whether training or coaching is better for sustaining results (e.g., van Buuren \& Edelenbos, 2013). Therefore, a critical factor associated with maintaining the effects of a well-being training program may be to expand the singular training focus.

Rather than focusing on one or two key well-being skills, it may be more important for efforts designed to enhance health and well-being to emphasize first responders' broader psychological skills (e.g., Blumberg \& Papazoglou, 2018; Blumberg, Schlosser, Papazoglou, Creighton, \& Kaye, 2019). Instead of learning a specific technique, the goal is to develop the skills that promote and maintain healthy functioning in many domains (i.e., physical, mental, emotional, social, spiritual, occupational, and financial). According to the California Commission on Peace Officer Standards and Training (2014), for example, "very effective" police performance involves maintaining competence in many psychological areas, including social competence, teamwork, adaptability/flexibility, conscientiousness/dependability, impulse control, decision-making/judgment, emotional regulation/ stress tolerance, assertiveness/persuasiveness, and integrity/ ethics (p. 51). Therefore, once first responders' well-being is operationalized, one recognizes the importance of focusing on a variety of competencies when designing a training tool intended to have long-term benefits.

\section{The HEROES Project}

One such broad-based training program is The HEROES Project (Thornton, Blumberg, Papazoglou, \& Giromini, 2020). The HEROES Project is a six-lesson online course taken over eight weeks "that combines the therapeutic tools of clinical and organizational psychology and provides first responders access to a self-driven well-being program" (p. 155). The theme for each of the six lessons corresponds to the letters in the title: Hope, Efficacy, Resilience, Optimism, Empathy, and Socialization. These skills are rooted in the theory of psychological capital (PsyCap), which recognizes that healthy employees are "an indispensable asset" in every organization
(Luthans \& Youssef, 2004, p. 143). The HEROES course uses active-learning techniques and provides participants with practical, job-related well-being strategies:

Participants start a project in lesson one and continue it throughout the training. They a re then encouraged after conclusion of the training to regularly update it over the course of their careers. Each training activity adds an essential element to the project, challenging trainees to apply lessons to their own lives. The training is self-paced. Trainees are able to take as much time as they want to critically evaluate and apply the learning outcomes. Each lesson includes a video lecture delivered by the project author [the present paper's fourth author] with downloadable lecture notes focused on learning outcomes. Trainees are virtually introduced to Mitch Kazjer, a police officer who survived being shot on the job, and are provided with short video clips throughout the training, wherein he details his journey to resilience. Additional resources such as podcasts, books, and articles that are particularly relevant to the development of resilience are made available to the trainees as adjuncts to the lesson materials. At the conclusion of the training program, first responders receive a certificate of achievement. (p. 157)

\section{The Present Study}

The present study was initiated to obtain results from participants who volunteered to complete The HEROES Project. The empirical question was: How are first responders impacted by completing the course? Specifically, because of the recognized incidence among first responders of stress and trauma reactions and anxiety and depression symptoms, and, conversely, the known benefits of positive psychological capital, these were the variables assessed. Secondarily, and, perhaps most importantly when it comes to first responders' health and well-being, did the benefits of the training last? The goal was to identify whether this self-paced, off-duty program provided first responders with the tools to stay healthy despite some of the more toxic stressors of their jobs. Thus, data was collected at five separate times up to two years after completion of the program. The study received approval from the Institutional Review Board of the fourth author's prior university.

\section{METHODS}

\section{Participants}

Volunteers were recruited from the police and fire departments of a medium-sized city in the Midwestern United States. In all, 124 participants (116 males and 8 females) completed the training program. Most participants completed all assessment components of the study $(100 \%$ at T2; $93 \%$ at T3; $90 \%$ at T4; and, $89 \%$ at T5). Although the sample consisted of 55 police officers $(44 \%)$ and 69 firefighters (56\%), no differences were found between the two groups, so all analyses reflect the combined group of "first responders." The age breakdown of participants was: $18-29=9 ; 30-44=60 ; 45-59=52 ; 60+=3$. Years of service of the participants was: $0-5=8 ; 6-10=22 ; 11-15=22 ; 16-20=$ $30 ; 21-25=18 ; 26+=24$. To determine whether two additional factors other than completion of the course had an influence 
on the outcome measures, participants were also asked about the frequency of their physical activity outside of work and if they "lived an active life of faith."

\section{Measures}

Participants were asked to complete three self-report instruments related, respectively, to their: 1) psychological capital (i.e., well-being); 2) experience of post-traumatic stress symptoms; and, 3) levels of depression, anxiety, and stress.

$P C Q-24$. The Psychological Capital Questionnaire 24 (Luthans, Avolio, Avey, \& Norman, 2007) is a 24-item selfreport instrument that measures dimensions, which the authors refer to as positive PsyCap. Specifically, the PCQ assesses the extent to which participants feel self-efficacy, hope, optimism, and resilience on a five-point Likert scale from strongly disagree to strongly agree. The authors reported a Cronbach's alpha coefficient score of 0.91 for the scale.

PCL-5-Civilian Version. The DSM V PTSD ChecklistCivilian Version is a 20-item self-report checklist designed to evaluate symptomology of post-traumatic stress disorder (PTSD). The civilian version is used to evaluate responses to traumatic situations encountered in the course of civilian life. Using a five-point Likert scale, respondents indicate the degree of impact a traumatic event has had on their emotional state. Originally created by Weathers, Huska, and Keane (Weathers, Litz, Herman, Huska, \& Keane, 1991; 1993), the scale was updated by the National Center for PTSD in 2014.

DASS-21. The Depression, Anxiety, Stress Scale (DASS21 ) is a 21-item self-report instrument that measures the severity or frequency of feelings of depression, anxiety, and stress in a non-clinical sample (Lovibond \& Lovibond, 1995). It has been used in over 7,000 studies involving police and firefighters (Thoreau Walden University Discovery Service).

\section{PROCEDURES}

Participants completed the three measures at Time 1 (prior to beginning the training), at the conclusion of the eight-week program (Time 2), and again at one year after the initial assessment (Time 3). Additionally, participants completed only the PCL and PCQ at 18 months (Time 4) and again two years after the initial assessment (Time 5). The measures were delivered via the Survey Monkey platform to each participant's personal e-mail address at each data collection point. However, due to a technical glitch on Survey Monkey, the distribution of the DASS to participants at T4 was corrupted, so that measure was not administered then or at T5. Between $\mathrm{T} 1$ and T2, each participant completed the online psychological skills development course, details of which can be found in a recent book chapter (Thornton et al., 2020)

\section{RESULTS}

The goal of the current study was to evaluate the impact of The HEROES Project on scores of psychological trauma, depression, anxiety, stress, and well-being. We performed a series of repeated-measures ANOVAs investigating whether the scores of PCL-5, DASS (depression, anxiety, and stress), and $P C Q$ (hope, optimism, self-efficacy, and resilience) varied across time. To deepen our understanding of the phenomenon, we explored the extent to which the effectiveness of the training could be moderated by factors such as age of the trainee, baseline levels of distress, and PsyCap, etc. Therefore, a series of mixed ANOVAs were computed.

Impact of Training on Psychological Distress and PsyCap Table I presents descriptive statistics and Cronbach's alphas for all key measures under investigation. A first consideration that deserves mentioning is that PCL-5, DASS Depression, DASS Anxiety, DASS Stress, and PCQ Total produced Cronbach's alpha values $\geq .68$ in almost all cases, with the sole exception of DASS Anxiety, which produced a value of .49 at T3. Conversely, each of the four subscales of the PCQ produced unsatisfactory alpha values (i.e., <.70; Nunnally, 1978 ) in at least one the five data points. Accordingly, the following analyses on PCQ scores focused on the total score only, and disregarded the subscale scores.

TABLE I

Cronbach's alphas and descriptive statistics.

\begin{tabular}{|c|c|c|c|c|c|c|c|c|c|c|c|c|c|c|c|}
\hline & & $\begin{array}{c}T 1 \\
N=12\end{array}$ & & & $\begin{array}{c}\mathrm{T} 2 \\
N=124\end{array}$ & & & $\begin{array}{c}\text { T3 } \\
N=116\end{array}$ & & & $\begin{array}{c}\mathrm{T} 4 \\
\mathrm{~N}=112\end{array}$ & & & $\begin{array}{c}\text { T5 } \\
N=110\end{array}$ & \\
\hline & $\alpha$ & $M$ & SD & $\alpha$ & $M$ & SD & $\alpha$ & $M$ & SD & $\alpha$ & $M$ & SD & $\alpha$ & M & SD \\
\hline PCL-5 & .96 & 16.8 & 14.7 & .90 & 6.9 & 6.8 & .84 & 5.0 & 4.5 & .77 & 4.4 & 3.6 & .71 & 4.1 & 3.1 \\
\hline DASS Depression & .90 & 9.2 & 9.3 & .80 & 4.0 & 5.3 & .74 & 2.6 & 3.6 & - & - & - & - & - & - \\
\hline DASS Anxiety & .79 & 5.1 & 6.1 & .69 & 2.1 & 3.4 & .49 & 1.4 & 2.2 & - & - & - & - & - & - \\
\hline DASS Stress & .86 & 13.5 & 9.5 & .73 & 6.0 & 5.8 & .68 & 4.1 & 4.1 & - & - & - & - & - & - \\
\hline PsyCap Hope & .97 & 23.7 & 9.6 & .79 & 28.8 & 3.2 & .41 & 32.0 & 1.8 & .35 & 32.4 & 1.4 & .64 & 33.4 & 1.8 \\
\hline PsyCap Optimism & .97 & 23.4 & 9.7 & .71 & 31.6 & 2.6 & .26 & 32.3 & 1.6 & .37 & 32.6 & 1.5 & .16 & 32.4 & 1.4 \\
\hline PsyCap Self-Efficacy & .97 & 23.8 & 9.2 & .86 & 30.9 & 3.5 & .66 & 32.4 & 2.0 & .64 & 32.6 & 1.8 & .49 & 33.5 & 1.7 \\
\hline PsyCap Resilience & .97 & 23.5 & 9.1 & .82 & 31.7 & 3.0 & .41 & 32.4 & 1.7 & .52 & 32.7 & 1.6 & .70 & 33.6 & 1.8 \\
\hline PsyCap Total & .99 & 94.4 & 36.7 & .93 & 123.0 & 10.9 & .71 & 129.1 & 4.9 & .76 & 130.2 & 4.6 & .80 & 132.9 & 5.2 \\
\hline
\end{tabular}

DASS $=$ depression, anxiety, and stress scale; PsyCap = psychological capital. 
Noteworthy, Cronbach's alphas tended to decrease when going from $\mathrm{T} 1$ to T5. A possible explanation for this finding is that the variability of the scores (i.e., SD values) tended to decrease as well, when going from T1 to T5. Indeed, because Cronbach's alphas reflect the correlations among all possible split-halves of a given scale and since co-variation cannot occur without some variation, it is likely that alpha decreased from $\mathrm{T} 1$ to $\mathrm{T} 5$ simply because the scores tended to become more homogeneous from T1 to T5. Figures 1 and 2 show that the scores of PCL-5, DASS Depression, DASS Anxiety, DASS Stress, and $P C Q$ Total became increasingly homogeneous with the passage of time.

\section{Influence of Demographic and Behavioral Variables}

We next tested whether age ( $<45$ vs. $\geq 45)$, experience in the profession (0-10 years vs. 11-20 years vs. $21+$ years), faith (yes vs. no), or physical activity (0-3 times per week vs. 4+ times per week) had an impact on the effectiveness of the training. We tested a series of mixed ANOVAs, with Time as our within-subject factor (considering PCL-5, DASS Depression, DASS Anxiety, DASS Stress, and PCQ Total scores), and each of the demographic and behavioral variables described above, entered one at a time, as our betweensubject factors. Of the 20 interaction effects under investigation, none resulted in statistical significance after applying Bonferroni correction.

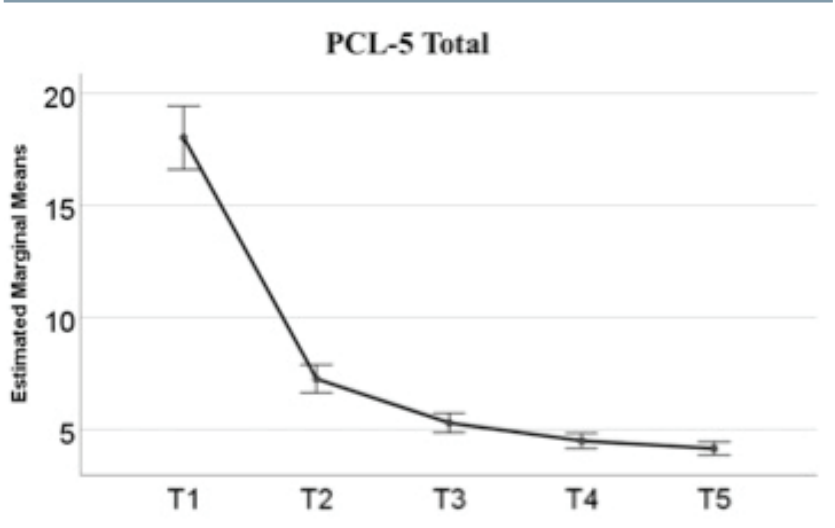

PCQ Total

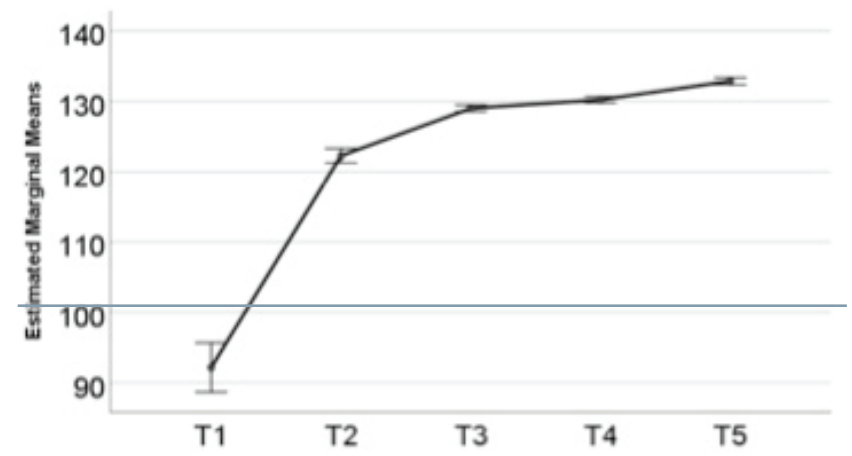

FIGURE 1 Graphical representation of PCL-5 and PCQ Total Scores. Error bars represent the standard error of the mean. $P C Q=$ Psychological Capital Questionnaire.

\section{Influence of Psychological Distress and Resources at the Baseline}

Lastly, we investigated whether the baseline levels of psychological distress (i.e., PCL-5 and DASS scores) and resources (i.e., $P C Q$ scores) were impacted by the effectiveness of the training. As a proxy marker of the levels of psychological distress at baseline, we calculated the $z$ average of the T1 scores of PCL-5, DASS Depression, DASS Anxiety, and DASS StresS (which correlated with each other at $r \geq .63, p<.01$ ). Individuals with a $z$ score greater than zero were considered to be at a high level of distress, and individuals with a z score lower than zero were considered to be at a low level of distress. For the psychological resources, individuals with a T1 score on PCQ Total greater than the median (i.e., PCQ Total $=115$ ) were considered to have good psychological resources while individuals with a T1 PCQ Total score lower than the median were considered to have poor psychological resources.

For the PCL-5 scores, a mixed 2 (between-subjects factor, baseline level of distress: low vs. high) by 2 (between-subjects factor, baseline level of resources: poor vs. good) by 5 (withinsubjects factor, time: T1 vs. T2 vs. T3 vs. T4 vs. T5) ANOVA revealed a statistically significant interaction between time and baseline level of distress, $F(4,424)=86.4, p<.01$ (Table II). No other interaction factors were statistically significant, $p \geq$ .12. As shown in Figure 3, the training had a greater, positive impact on individuals who had higher levels of distress at baseline. The size of the difference from T1 to T5 in PCL-5
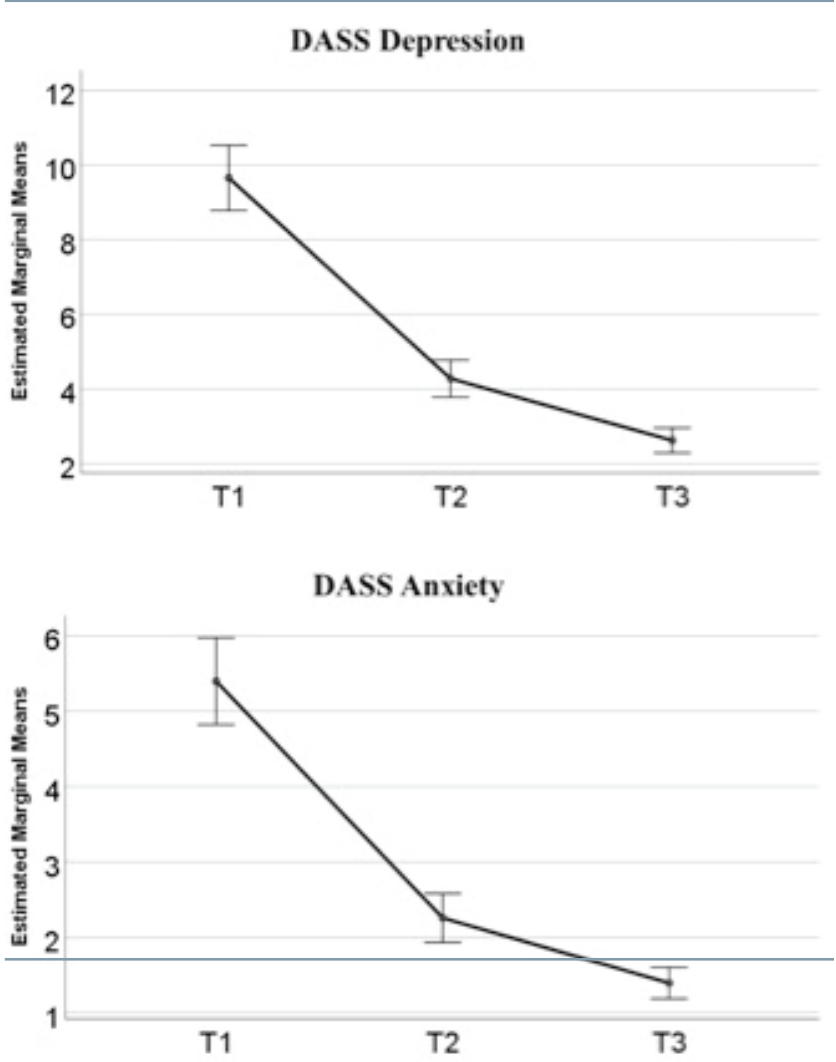

FIGURE 2 Graphical Representation of DASS Scores. Error bars represent the standard error of the mean. DASS = depression, anxiety, and stress scale. 
TABLE II Results of repeated-measures ANOVAs.

\begin{tabular}{|c|c|c|c|c|c|c|}
\hline & \multirow[t]{2}{*}{ Main effect } & \multirow[t]{2}{*}{ Pairwise Comparisons } & \multicolumn{4}{|c|}{ Cohen's $d$ Effect Size } \\
\hline & & & T1 vs. T2 & T1 vs. T3 & T1 vs. T4 & T1 vs. T5 \\
\hline PCL-5 & $F(4,436)=132.8, p<.01$ & All p's $<.01$ & .86 & 1.07 & 1.13 & 1.16 \\
\hline DASS Depression & $F(2,230)=134.0, p<.01$ & All $p^{\prime} s<.01$ & .69 & .92 & - & - \\
\hline DASS Anxiety & $F(2,230)=81.6, p<.01$ & All $p^{\prime} s<.01$ & .61 & .80 & - & - \\
\hline DASS Stress & $F(2,230)=237.4, p<.01$ & All $p^{\prime} s<.01$ & .95 & 1.27 & - & - \\
\hline PCQ Total & $F(4,436)=133.6, p<.01$ & All $p^{\prime} s<.01$ & 1.06 & 1.30 & 1.34 & 1.43 \\
\hline
\end{tabular}

DASS = depression, anxiety, and stress scale; $P C Q=$ Psychological Capital Questionnaire.

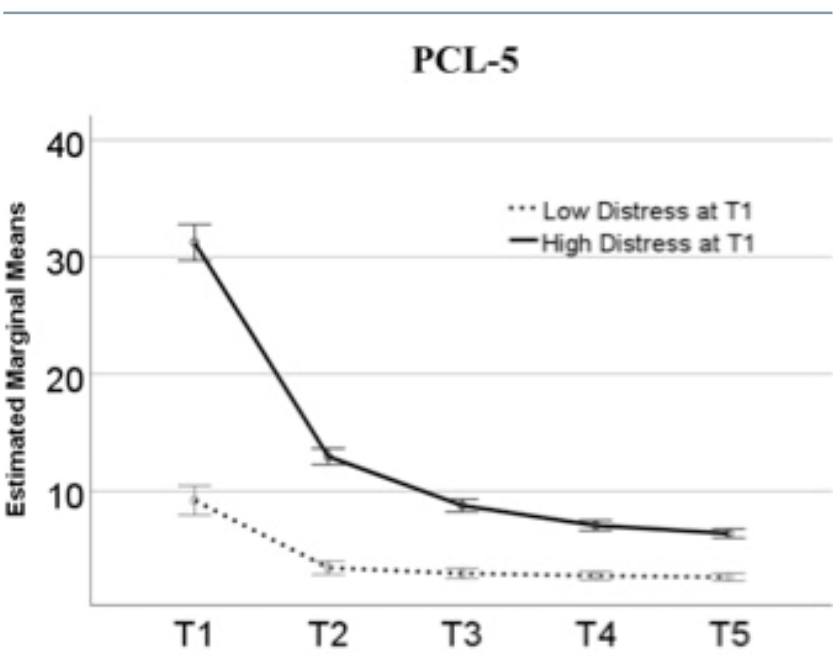

FIGURE 3 Graphical Representation of PCL-5 Scores. Error bars represent the standard error of the mean.

scores for individuals with a high distress level at baseline consisted of a remarkably large Cohen's $d$ value of 2.33. A similar pattern was observed when inspecting the impact of baseline levels of distress and resources on DASS and PCQ scores. It should be noted also that baseline level psychological resources influenced the extent to which the training increased the scores of PCQ Total. More specifically, the higher the distress and the lower the psychological resources were at T1, the greater was the impact of the training on the participants' psychological resources.

\section{DISCUSSION}

The results from the present study were very robust. After completing the program, participants reported significantly reduced stress, depression, anxiety, and trauma symptoms and significantly higher levels of psychological capital. These results were even greater for participants whose pre-program scores reflected greater psychological distress and lower psychological resources. It could be said that The HEROES Project worked best for those first responders who needed it the most.

Of great significance was that the impact of the psychological skills training program endured through the two-year assessment point. Moreover, there was some indication that participants continued to improve past the conclusion of the program. It appears that the skills developed during the eight-week training were used long after participants ended the program. Thus, rather than learning a specific skill, The HEROES Project instilled psychological skills and methods to practice those skills in a manner that led to sustained psychological well-being. This was independent of participants' ages, years in their profession, level of physical activity, and extent of faith activities.

The HEROES Project reinforces the theory that efforts to improve first responder well-being should emphasize broad-based psychological skill development. To maintain well-being, first responders require a diverse skill set, because threats to their well-being are multidimensional (e.g., Kwiatkowski \& Robison, 2020; Thornton, 2020). Therefore, wellbeing programs should expand to address the many areas that pose a risk to first responders' health and well-being.

\section{Limitations}

The present study has some limitations and, as mentioned, was a rather narrow preliminary study. The sample size was small, geographically constrained, contained an underrepresented number of female participants, and did not collect information about the ethnic or racial background of the participants. All of these factors may limit the generalizability of the findings. Although the present sample contained participants of various ages and years of experience, it cannot be ignored that there may have been a self-selection effect whereby those who volunteered were more likely to benefit from the training than those who did not choose to participate. Finally, although unlikely, the possible impact of practice effects cannot be overlooked. However, the time between administration of the measures and the continued improvement of participants' scores can provide some confidence that a practice effect was not responsible for the scores that were obtained.

\section{Future Directions}

The efficacy of The HEROES Project remains to be tested more rigorously. A significantly larger number of participants from broad geographical areas should be assessed. The program should be taken by more females as well as by first responders of all cultural backgrounds to further determine the impact of this training on various demographic groups. It may be advisable to employ a variety of assessment measures to avoid practice effects. 
Nevertheless, as a pilot study, the present findings offer some encouragement that The HEROES Project may be an important option to boost first responders' well-being. It is significant that the present results demonstrated that the program benefited participants who were relatively healthy and that it had an even better impact on first responders who were not functioning as well before the training. This indicates that The HEROES Project could serve both as an adjunct to clinical interventions and as a preventive tool for first responders who have not yet begun to experience the most deleterious effects of their job. Nevertheless, while more research is necessary before making any definitive conclusions, the present findings provide a promising start.

\section{ACKNOWLEDGMENTS}

The authors would like to express their gratitude to all first responders who devoted their time to participate in this study. In addition, the authors would like to thank Mr. Prashant Aukhojee, HBSc, research assistant, who volunteered his time to assist the authors with the final formatting and proper citation of this manuscript.

\section{CONFLICT OF INTEREST DISCLOSURES}

The authors declare that there are no conflicts of interest.

\section{AUTHOR AFFILIATIONS}

${ }^{*}$ California School of Professional Psychology, Alliant International University, San Diego, CA, USA

${ }^{\dagger}$ Department of Psychology, University of Turin, Turin, Italy

‡Yale School of Medicine, New Haven, CT, USA

§Kelley School of Business, Indiana University, Bloomington, IN, USA

\section{REFERENCES}

Biebel, C. J. (2010). Analysis of variables affecting participant success within a law enforcement wellbeing program. Dissertation Abstracts International: Section B: The Sciences and Engineering. ProQuest Information \& Learning.

Blumberg, D. M., \& Papazoglou, K. (2018, July). Arming police officers with psychological survival strategies. The ePolice Chief, 12-13 https://www.policechiefmagazine.org/osc-psych-survivalstrategies/.

Blumberg, D., Schlosser, M. D., Papazoglou, K., Creighton, S., \& Kaye, C. C. (2019). New directions in police academy training: A call to action. International Journal of Environmental Research and Public Health, 16(24), 4941. https://doi.org/10.3390/ ijerph16244941

California Commission on Peace Officer Standards and Training. (2014). Peace officer psychological screening manual. California Commission on Peace Officer Standards and Training (2014). Peace Officer Psychological Screening Manual. Sacramento, CA: California POST.

Carpenter, G. S. J., Carpenter, T. P., Kimbrel, N. A., Flynn, E. J., Pennington, M. L., Cammarata, C., ... Gulliver, S. B. (2015). Social support, stress, and suicidal ideation in professional firefighters. American Journal of Health Behavior, 39121, 191-196. https://doi.org/ 10.5993/AJHB.39.2.5

Chitra, T., \& Karunanidhi, S. (2018). The impact of resilience training on occupational stress, resilience, job satisfaction, and psychological well-being of female police officers. Journal of Police and Criminal Psychology. https://doi.org/10.1007/s1 1896-018-9294-9

Christopher, M. S., Hunsinger, M., Goerling, L. R. J., Bowen, S., Rogers,
B. S., Gross, C. R., ... Pruessner, J. C. (2018). Mindfulness-based resilience training to reduce health risk, stress reactivity, and aggression among law enforcement officers: A feasibility and preliminary efficacy trial. Psychiatry Research, 264, 104-115. https://doi. org/10.1016/i.psychres.2018.03.059

Creighton, S., \& Blumberg, D. M. (2016). Officer wellbeing is fundamental to officer safety: The San Diego Model. In Police Executive Research Forum, Critical Issues in Policing Series: Guiding Principles in Use of Force. Washington, DC.

Hibbard, J. H., \& Greene, J. (2014). The impact of an incentive on the use of an online self-directed wellness and self-management program. Journal of Medical Internet Research, 16(10), e217. https://doi. org/10.2196/jmir.3239

Kuehl, H., Mabry, L., Elliot, D. L., Kuehl, K. S., \& Favorite, K. C. (2013). Factors in adoption of a fire department wellbeing program: Champand-chief model. Journal of Occupational and Environmental Medicine, 55(4), 424-429.

Kwiatkowski, C. C., \& Robison, A. J. (2020). Internal threats to police wellbeing. In K. Papazoglou \& D. M. Blumberg (Eds.), POWER: Police officer wellbeing, ethics, \& resilience (pp. 29-36). Cambridge, MA: Academic Press.

Lovibond, P. F., \& Lovibond, S. H. (1995). The structure of negative emotional states: Comparison of the Depression Anxiety Stress Scales (DASS) with the Beck Depression and Anxiety Inventories. Behaviour Research and Therapy, 33(3), 335-343. https://doi. org/10.1016/0005-7967(94)00075-U

Luthans, F., Avolio, B. J., Avey, J. B., \& Norman, S. M. (2007). Positive psychological capital: Measurement and relationship with performance and satisfaction. Personnel Psychology, 60131, 541-572. https:// doi.org/10.1111/j.1744-6570.2007.00083.x

Luthans, F., \& Youssef, C. M. (2004). Human, social, and now positive psychological capital management: Investing in people for competitive advantage. Organizational Dynamics, 33(2), 143-160. https:// doi.org/10.1016/i.orgdyn.2004.01.003

Mumford, E. A., Taylor, B. G., \& Kubu, B. (2015). Law enforcement officer safety and wellness. Police Quarterly, 18(2), 111-133. https://doi.org/ $10.1177 / 1098611114559037$

Nunnally, J. C. (1978). Phychometric theory. In Psychometric theory. New York, NY: McGraw-Hill.

Papazoglou, K., \& Blumberg, D. M. (Eds.). (2020). Power: Police officer wellbeing, ethics, \& resilience. Cambridge, MA: Academic Press.

Smith, B. W., Ortiz, J. A., Steffen, L. E., Tooley, E. M., Wiggins, K. T., Yeater, E. A., ... Bernard, M. L. (2011). Mindfulness is associated with fewer PTSD symptoms, depressive symptoms, physical symptoms, and alcohol problems in urban firefighters. Journal of Consulting and Clinical Psychology, 79(5), 613-617. https://doi.org/10.1037/ a0025189

Song, Z., \& Baicker, K. (2019). Effect of a workplace wellness program on employee health and economic outcomes. JAMA, 321(15), 1491. https://doi.org/10.1001/jama.2019.3307

Thoen, M. A., Dodson, L. E., Manzo, G., Piña-Watson, B., \& Trejos-Castillo, E. (2019). Agency-offered and officer-utilized suicide prevention and wellness programs: A national study. Psychological Services. https:// doi.org/10.1037/ser0000355

Thornton, A. R. (2020). Police officer wellbeing. In K. Papazoglou \& D. M. Blumberg (Eds.), Power: Police officer wellbeing, ethics, \& resilience (pp. 7-27). Cambridge, MA: Academic Press.

Thornton, A. R., Blumberg, D. M., Papazoglou, K., \& Giromini, L. (2020). The HEROES Project: Building mental resilience in first responders. In C. Bowers, D. Beidel, \& M. Marks (Eds.), Mental Health 
Intervention and Treatment of First Responders and Emergency Workers (pp. 154-168). https://doi.org/10.4018/978-1-5225-9803-9. ch009

Tuttle, B. M., Blumberg, D. M., \& Papazoglou, K. (2019). Critical challenges to police officer wellness. In H. Pontell (Ed.), Oxford research encyclopedia of criminology and criminal justice. https://doi.org/10.1093/ acrefore/9780190264079.013.538

van Buuren, A., \& Edelenbos, J. (2013). Organizational competence development in two public agencies in the Netherlands. Public
Personnel Management, 42(3), 385-402. https://doi.org/10.1177/ 0091026013495771

Weathers, F. W., Litz, B. T., Herman, D., Huska, J., \& Keane, T. (1991). PTSD Checklist - Civilian Version (PCL-C). In National Center for PTSD. Boston, MA.

Weathers, F., Litz, B., Herman, D., Huska, J., \& Keane, T. (1993). The PTSD Checklist (PCL): Reliability, validity, and diagnostic utility. Annual Convention of the International Society for Traumatic Stress Studies. San Antonio, TX. 